\title{
Muséologies
}

Les cahiers d'études supérieures

muséologies

\section{La réactualisation des collections fermées, trois études de cas. Les collections du Isabella Stewart Gardner Museum, de Kettle's Yard et de la New Art Gallery Walsall}

\author{
Justine Lebeau
}

Volume 4, numéro 1, automne 2009

URI : https://id.erudit.org/iderudit/1033532ar

DOI : https://doi.org/10.7202/1033532ar

Aller au sommaire du numéro

Éditeur(s)

Association Québécoise de Promotion des Recherches Étudiantes en Muséologie (AQPREM)

ISSN

1718-5181 (imprimé)

1929-7815 (numérique)

Découvrir la revue

Citer cet article

Lebeau, J. (2009). La réactualisation des collections fermées, trois études de cas. Les collections du Isabella Stewart Gardner Museum, de Kettle's Yard et de la New Art Gallery Walsall. Muséologies, 4(1), 64-77.

https://doi.org/10.7202/1033532ar
Résumé de l'article

Les collections fermées, conservées dans des demeures historiques, posent de véritables défis de renouvellement et d'actualisation à ses dirigeants. Ceux-ci doivent répondre aux nouvelles exigences des visiteurs en matière d'accueil et de visites muséales, tout en respectant leur devoir d'immuabilité envers les dispositions d'exposition de leurs collections. À partir de trois études de cas, soit les collections du Isabella Stewart Gardner Museum, de Kettle's Yard et de la New Art Gallery Walsall, Justine Lebeau détaille quatre stratégies de réactualisation de ce type de collections adoptées par ces institutions.
Tous droits réservés @ muséologies Les cahiers d'études supérieures, 2009
Ce document est protégé par la loi sur le droit d'auteur. L'utilisation des services d'Érudit (y compris la reproduction) est assujettie à sa politique d'utilisation que vous pouvez consulter en ligne.

https://apropos.erudit.org/fr/usagers/politique-dutilisation/ 


\section{Article trois}

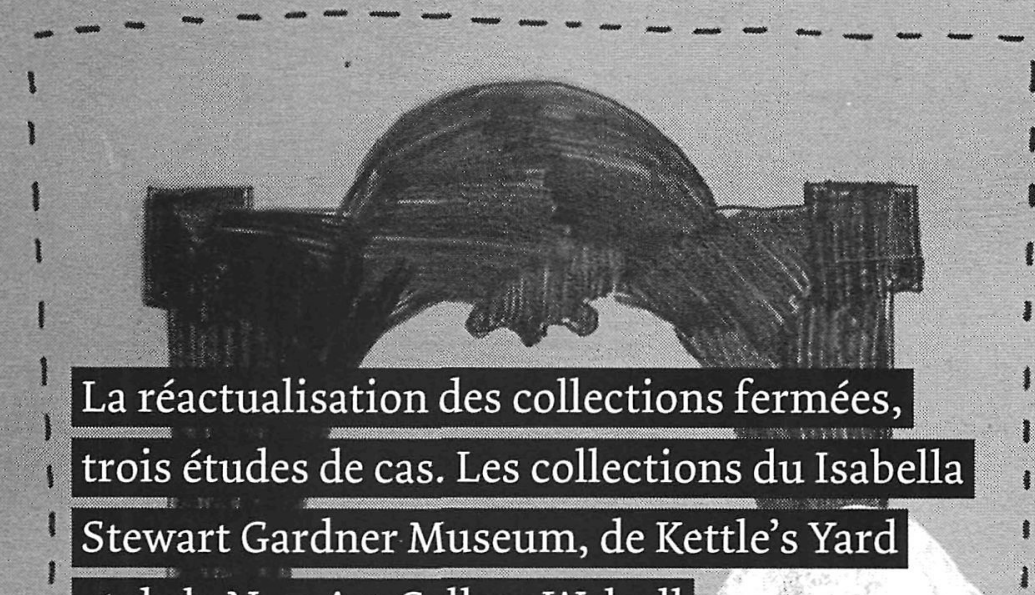

, et de la New Art Gallery Walsall

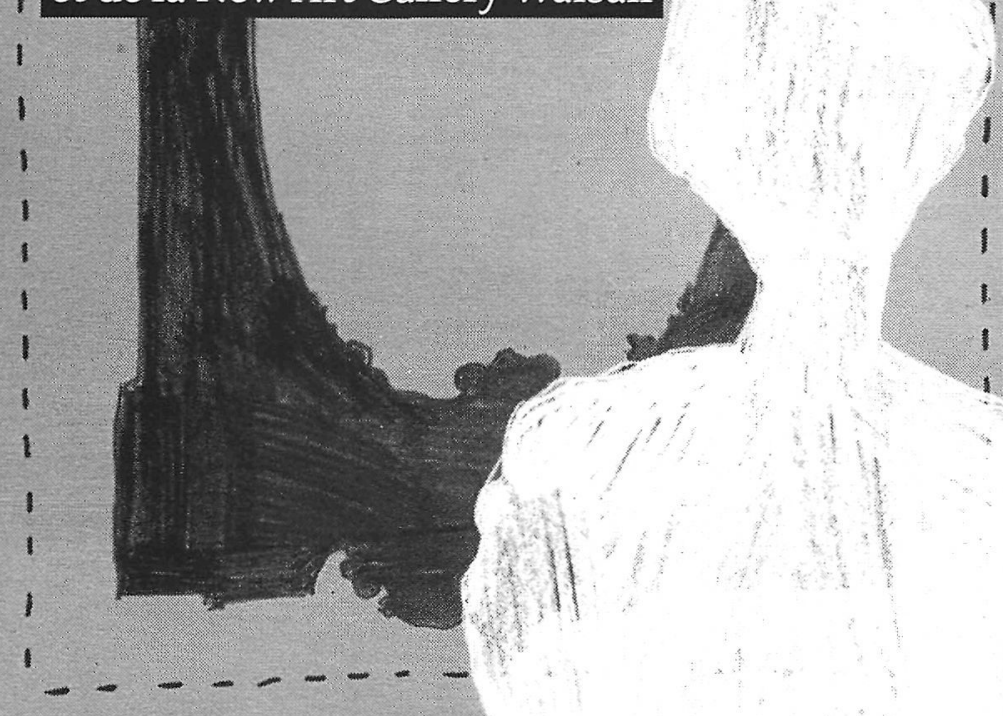


Les collections fermées, conservées dans des demeures historiques, posent de véritables défis de renouvellement et d'actualisation à ses dirigeants. Ceux-ci doivent répondre aux nouvelles exigences des visiteurs en matière d'accueil et de visites muséales, tout en respectant leur devoir d'immuabilité envers les dispositions d'exposition de leurs collections. À partir de trois études de cas, soit les collections du Isabella Stewart Gardner Museum, de Kettle's Yard et de la New Art Gallery Walsall, Justine Lebeau détaille quatre stratégies de réactualisation de ce type de collections adoptées par ces institutions.

JUSTINE LEBEAU EST ÉTUDIANTE À LA MAITTRISE EN ÉTUDES DES ARTS À L'UNIVERSITÉ DU QUÉBEC À MONTRÉAL. SON MÉMOIRE ANALYSE LES STRATÉGIES VISANT LA RÉACTUALISATION DES COLLECTIONS FERMÉES. ELLE TRAVAILLE PRÉSENTEMENT DANS UN GROUPE DE RECHERCHE SUR LA RÉACTUALISATION, RÉEXPOSITION ET PÉRENNITÉ DE L'ART CONTEMPORAIN, DONT LA CHERCHEURE PRINCIPALE EST FRANCINE COUTURE DE L'UNIVERSITÉ DU QUÉBEC À MONTRÉAL. ELLE A PAR AILLEURS PARTICIPÉ À LA RECHERCHE DOCUMENTAIRE DERRIÈRE L'EXPOSITION D'EEUVRES CONCEPTUELLES DE BILL VAZAN, WALKING INTO THE VANISHING POINT, À LA GALERIE VOX DE MONTRÉAL EN 2007. [justinelebeau@gmail.com] 
[1]

Le terme demeure historique-musée est tiré du DEMHIST, comité visant la gestion et la conservation de ces endroits depuis 1999 et qui est relié au Conseil international des musées (ICOM).

Pour des renseignements complémentaires, voir l'onglet About DEMHIST sur leur site Internet:<http://demhist.icom. museum/aboutdemhist.htm $>$

(consulté en avril 2009).

[2]

FREUDENHEIM, Tom «Installation Ruminations II: Comfort and Joy". Curator, vol. $51, n^{\circ} 2,2008$, p. 151-156.

[3]

Nous nuançons grandement ces caractérisations puisque, tel que nous le verrons plus tard, deux de nos études de cas sont à l'extérieur de ce cadre précis.

[4]

POMIAN, Krzysztof. Collectionneurs, amateurs et curieux. Paris, Venise $X V^{k}-X V I^{e}$ siècle. Paris: Gallimard, coll. «Bibliothèque des histoires*, p. 300-301.

[5]

DUNCAN, Carole. «Something Eternal: The Donor Memorial». In. DUNCAN, Carole. Civilizing Rituals, Inside Public Art Museums. Londres et New York: Routledge, 1995, p. 153, note infra $n^{\circ} 25$.
Depuis la fin de la décennie 1990, nous assistons à un intérêt renouvelé par rapport à la recherche et l'analyse des demeures historiques-musées ${ }^{[1]}$ et à la mise en évidence du besoin d'élaborer une terminologie appropriée afin d'en différencier les catégories et d'agglomérer les déterminations communes.

C'est dans le contexte muséal en perpétuelle transformation depuis le $\mathrm{XX}^{\mathrm{e}}$ siècle qu'il devient intéressant de considérer ces collections personnelles qui ont conservé leur assemblage intact, ainsi que leur gestion actuelle. En effet, celles-ci sont caractérisées par un tiraillement entre un désir d'adaptation et une responsabilité d'éviter toute altération puisque leur disposition immuable est désormais considérée tout à la fois en tant que témoignage esthétique et document historique.

Premièrement, elles permettent d'entrevoir l'importance de plus en plus grande attribuée aux événements temporaires au détriment des collections permanentes des musées. Deuxièmement, la tendance vers l'homogénéisation de la présentation muséale débutée au $\mathrm{XX}^{\mathrm{e}}$ siècle et menant à l'espace régulé du white cube vient confirmer la pertinence de préserver ces collections fermées puisqu'elles témoignent de modes inhabituels d'expérimentation des œuvres d'art ${ }^{(2)}$. Cet article soumet une définition approfondie de ce que nous entendons par collection fermée, une énumération de ses caractéristiques constituantes ainsi que l'analyse de quatre stratégies de réactualisation ayant été identifiées sur trois études de cas.

\section{La collection fermée}

La collection fermée est un ensemble d'objets hétéroclites, majoritairement constitué d'œuvres d'art, ayant été rassemblés par un ou plusieurs collectionneurs ou collectionneuses dans l'intention éventuelle de les dévoiler publiquement. Cette collection est ensuite léguée à une ville ou à une institution avec un lot de restrictions quant à la disposition spatiale et au maintien des objets qui la composent. Ces limitations testamentaires prohibent, 
par exemple, l'ajout ou le retrait d'œuvres, toute modification de l'assemblage des objets ou même l'exposition avec d'autres artefacts qui lui sont étrangers afin de conserver sa mise en scène originelle. La collection fermée est souvent impartie à même le bâtiment qu'il l'accueillait au départ, soit la résidence du collectionneur. Ce choix d'emplacement peut être expliqué en partie par l'expérience esthétique distincte du musée désirée par le donateur. Ce type de collection est habituellement issu de la fin du XIX ${ }^{e}$ siècle et est constitué par de riches industriels ou par le biais de fortunes familiales; on le retrouve aux États-Unis et dans plusieurs pays européens tels que la France, l'Angleterre, l'Italie et les Pays-Bas ${ }^{[3]}$.

Le terme collection fermée a été traduit de l'expression closed collection qualifiant ces collections permanentes dont les politiques d'acquisition sont arrêtées. Notre définition est redevable de plusieurs acceptions connexes, dont musée évergétique ${ }^{[4]}$ de Krzysztof Pomian, donor memorial ${ }^{[5]}$ de Carol Duncan, ainsi que plusieurs appellations du comité international des Demeures historiques-musées (DEMHIST), telles que les maisons de collectionneurs de Conny Bogaard ${ }^{[6]}$, la notion de collection intacte de Linda Young ${ }^{[7]}$ ainsi que le musée d'art personnel de FrederickJ. Fisher ${ }^{[8]}$. L'utilisation terminologique de collection fermée fait référence à la complétude de la collection (du moins dans l'esprit du collectionneur). À titre d'exemple, nous pensons aux collections fermées de la Barnes Foundation de Philadelphie, du musée Jacquemart-André de Paris, du musée Isabella Stewart Gardner de Boston, de la Freer Gallery of Art de Washington et, plus récemment, aux collections Kettle's Yard de Cambridge et Garman Ryan de la New Art Gallery Walsall.

Depuis le début des années 1990, nous assistons à un phénomène généralisé visant la réactualisation de ces collections fermées par le biais de stratégies déployées par les intervenants internes de ces institutions (dont conservateurs, directeurs, artistes). Celles-ci réévaluent de façon critique la portée narrative de la collection sans toutefois contrarier la disposition de ses assemblages. Nous décrirons maintenant quatre interventions esquivant astucieusement les limitations testamentaires des collectionneurs et ayant été extraites de l'analyse de trois collections fermées : le musée Isabella Stewart Gardner Museum, Kettle's Yard et la New Art Gallery Walsall.

Premièrement, nous discernons la construction adjacente ou le réaménagement interne de pavillons déjà existants qui ne sont pas ou plus réglementés par le testament. Deuxièmement, ces «nouveaux» espaces autorisent la manifestation d'expositions temporaires (historiques ou contemporaines) reliées thématiquement ou formellement à des aspects présents à même la collection permanente. Dans le même ordre d'idées, la troisième stratégie introduit des artistes en résidence dans l'institution et favorise le travail de création dialoguant avec la collection fermée. Enfin, l'accumulation d'œurres supplémentaires constituées en collections

\section{[6]}

Traduction libre de collector's house. Ce terme particulier comprend les demeures historiques-musées contenant une collection d'art rassemblée par un particulier et qui sont léguées dans l'intention de révéler un musée personnel public. Cette terminologie lui a paru primordiale pour ausculter huit exemples de demeures historiques-musées datées de 1870 à 1930 en Hollande aux Pays-Bas. (À ce sujet, voir : BOGAARD, Conny. "New Challenges for Dutch Collector's Houses *. In. PAVONI, Rosanna (dir.). New Forms of Management for Historic House Museums? Actes de la conférence annuelle du DEMHIST, Barcelone, 2-5 juillet 2001, 2002, p. 13-18; et BOGAARD, Conny, Marieke van VLIERDEN, Helen WÜSTEFELD et Kees van SHOOTEN. Huismusea in Nederland: Kasteel-Museum Sypesteyn en Het Ontstaan van Verzemelaarshuizen in Nederland (ca. 1870-1930) [Les demeures historiquesmusées aux Pays-Bas: Le château-musée Sypesteyn et les origines des maisons de collectionneurs aux Pays-Bas (ca 1870-1930) (traduction libre)]. Zwolle, Waanders, Loosdrecht: Van SypesteynStichting, 2007, 223 p.)

[7]

Traduction libre de la locution intact collection comprise par l'auteure en tant qu'assemblage conservé tel quel puisque sa signification est inhérente à sa disposition originelle. (YOUNG, Linda. «Is There a Museum in the House? Historic Houses as a Species of Museumm. Museum Management and Curatorship, vol. $22, n^{\circ} 1,2007, p .73$.) 
Traduction libre du terme art collector's personal museum. L'auteur conçoit cette nouvelle terminologie comme permettant, entre autres, la dénomination du musée Hillwood de Washington et, par extension, ces maisons contenant des collections privées d'art données dans l'intention de devenir publiques. (FISHER, Frederick J. "Change of Nomenclature: Clarity of Purpose». In. PAVONI, Rosanna (dir.). Historic House Museums as Witnesses of National and Local Identities.

Actes de la troisième conférence annuelle du comité DEMHIST, Amsterdam, 15-16 octobre 2002, 2003, p. 134.)

[9]

«Museum Founded by Mrs. J.L. Gardner». The New York Times, 23 juillet 1924, p. 4.

[10]

HIGONNET, Anne. «Private Museums, Public Leadership: Isabella Stewart Gardner and the Art of Cultural Authority». In. Cultural Leadership in America, Art Matronage and Patronage. Boston: Isabella Stewart Gardner Museum, vol. 27, p. 80.

[11]

Massachusetts Office of the Attorney General. Report of the Attorney for Fiscal Year Ending June 30, 1992. Publication $n^{\circ}$ 17464, 1992.<http://www. archive.org/stream/reportofattorney 1992mass/reportofattorney1992mass_djvu.txt> (consulté en janvier 2009).

[12]

Courriel de Pieranna Cavalchini daté du 15 décembre 2008.

[ 13]

EDGERS, Geoff. "Gardner Museum to Grow. 1903 Institution Plans Tripling Space in $1^{\text {st }}$ Major Expansion». The Boston Globe, 29 novembre 2004. <http:// www.boston.com/news/local/ articles/2004/11/29/gardner_ museum_to_grow $/$ ?page $=2 \mathrm{http}: / /$

www.boston.com/news/local/ articles/2004/11/29/gardner_museum_to_grow/?page $=2>$ (consulté en mars 2009). parallèles permet un ajustement indirect du contenu de la collection en comblant ses lacunes et en extrapolant certains aspects. Nos trois études de cas permettront l'analyse plus pointue d'une stratégie de réactualisation en particulier tout en présentant rapidement l'apport des autres.

\section{Le musée Isabella Stewart Gardner de Boston}

Le musée Gardner est la création d'Isabella Stewart Gardner (1840-1924) et il desservait de son vivant les fonctions contrastantes de résidence et d'environnement esthétique public. Le testament de 1924 transfere la propriété de l'entièreté du palais néo-vénitien et les œuvres d'art qui y sont distribuées à la ville de Boston et stipule que la moindre modification dans la disposition des objets de la collection entraînerait sa vente complète aux enchères afin de verser les fonds générés à l'Université Harvard ${ }^{[9]}$. La collection est déposée à l'intérieur de l'ancienne résidence d'Isabella Stewart Gardner et inclut des œuvres de divers artistes - Fra Angelico, Botticelli, Simone Martini, Michelangelo, Matisse, Degas, Titien, Rubens, Rembrandt, Raphæl, Turner - assemblées stratégiquement de pair avec un grand nombre d'objets d'art décoratifs, de livres anciens et de meubles. La scénographie des objets du musée Gardner date du début du XX $X^{\mathrm{e}}$ siècle alors qu'il était encore privé. En outre, près d'un siècle plus tard, l'exposition de ces objets reste la même et témoigne de l'idiosyncrasie de la propriétaire plutôt que d'un ordonnancement étudié ${ }^{[10]}$, et conserve, malgré l'adhésion à un musée public, son " aura privée».

Nonobstant les interdictions, le Gardner a procédé à l'ajout d'espaces limitrophes en 1933 afin d'accommoder ses employés. Ces zones précises ont ensuite été réaménagées dans la décennie $1970^{[11]}$ afin d'être transformées officiellement en 1992 en un espace d'exposition temporaire, un café et une boutique ${ }^{[12]}$. Le musée est toujours en cours de financement en vue de l'érection d'un pavillon contemporain contigu trois fois plus volumineux (45000 pieds carrés) que la construction de 1903 (15 000 pieds carrés) ${ }^{[13]}$. Ce futur édifice procédera à l'extraction et à l'accueil des programmes qui avaient été tant bien que mal intégrés à la demeure historique-musée, lui donnant l'occasion de recouvrer sa fonction unique d'exposition permanente.

C'est à l'intérieur de ces "nouveaux» espaces non réglementés que se trouve la deuxième intervention examinée: la mise en place d'expositions temporaires questionnant le caractère particulier de la collection en l'évaluant selon un regard critique, parfois en accord ou en opposition avec la pensée du collectionneur, mais respectant néanmoins l'inaltérabilité 


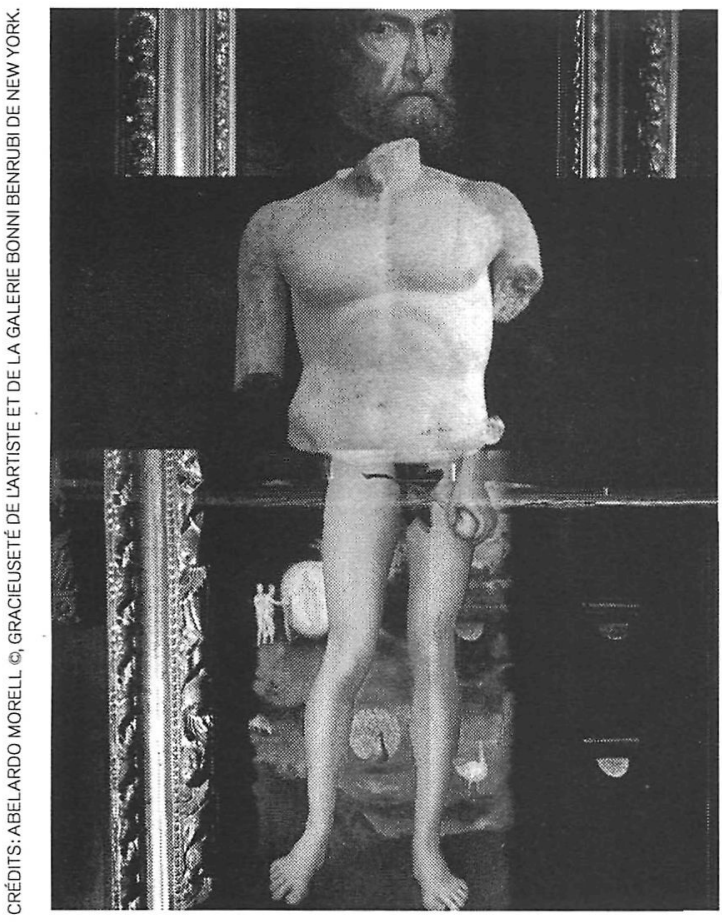

ILL. 1 Abelardo Morell, Exquisite Corpse, 1998.

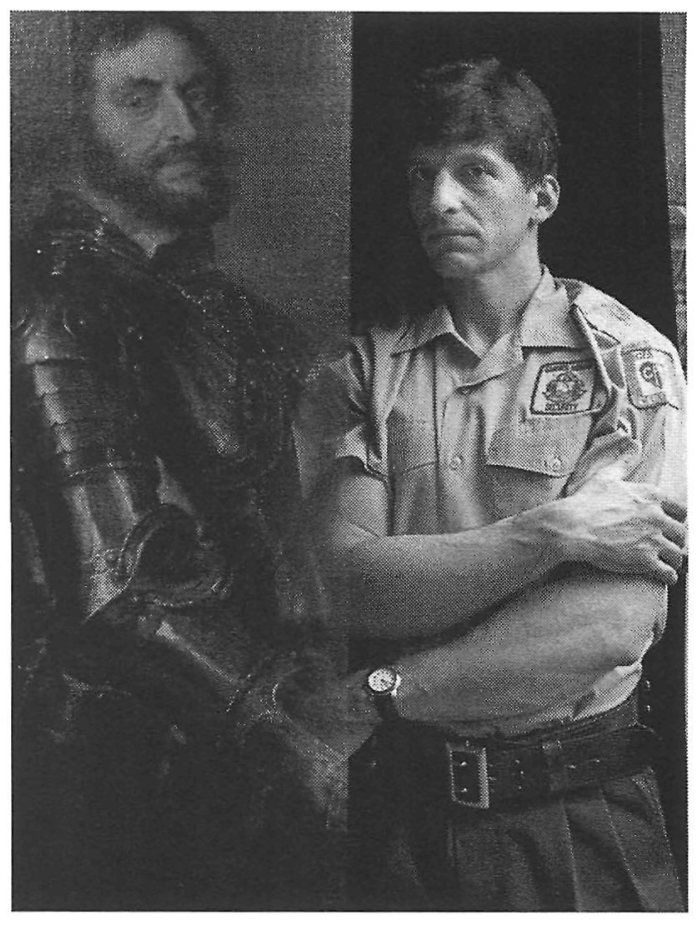

ILL. 2 Dave and the Earl of Arundel, 1998. physique des lieux. De telles expositions sont constituées en tant qu'extrapolations de la collection fermée afin d'en proposer un renouvellement par le biais de liens thématiques ou formels. En guise d'exemple, l'artiste cubain Abelardo Morell a été convié par le musée en 1998 à réaliser un projet relié aux problématiques du Gardner. Grâce à l'usage du médium photographique, l'artiste parvient à la juxtaposition d'œuvres ne possédant habituellement aucune proximité physique entre elles. Par exemple, Exquisite Corpse de 1998 (ill. 1) accole trois éléments disparates provenant de la collection fermée: la tête d'un vieil homme et les jambes de ce qui semble être une représentation d'Adam et, au centre de ce "cadavre exquis ", un torse en marbre relie les deux parties tronquées ${ }^{[14]}$, créant ainsi une image inédite.

Les créations photographiques de 1998, Tim and Rembrandt, Joan and Sir William Butts et David and the Earl of Arundel (ill. 2), réitèrent cette manœuvre en immortalisant des employés du musée (gardiens de sécurité, jardiniers, concierges, etc.) aux côtés d'œuvres précises, tissant ainsi des liens entre leur disposition statique évoquant un temps passé et la mouvance du travail issu du présent qui transforment simultanément les deux images ${ }^{[15]}$. Ces images ont été exposées dans l'espace voisin à la collection permanente

\section{[14]}

MORELL, Abelardo, Charles SIMIC, Jennifer R. GROSS et ISABELLA STEWART GARDNER MUSEUM. Abelardo Morell, Face to Face. Photographs at the Gardner Museum. Boston: The Museum, 1998 , p. 24.

[15]

Id., p. 25. 


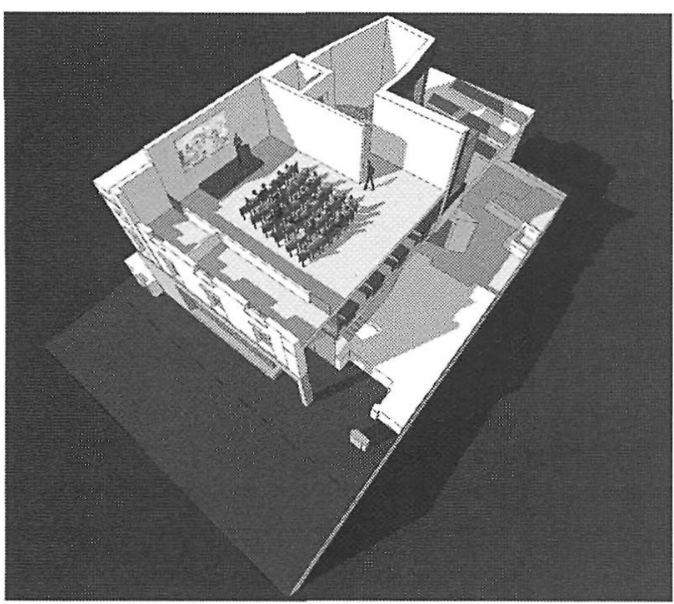

ILL. 4 Schéma de l'extension prévue pour Kettle's Yard par la firme d'architectes Jamie Fobert de Londres, vue de la salle de conférence.

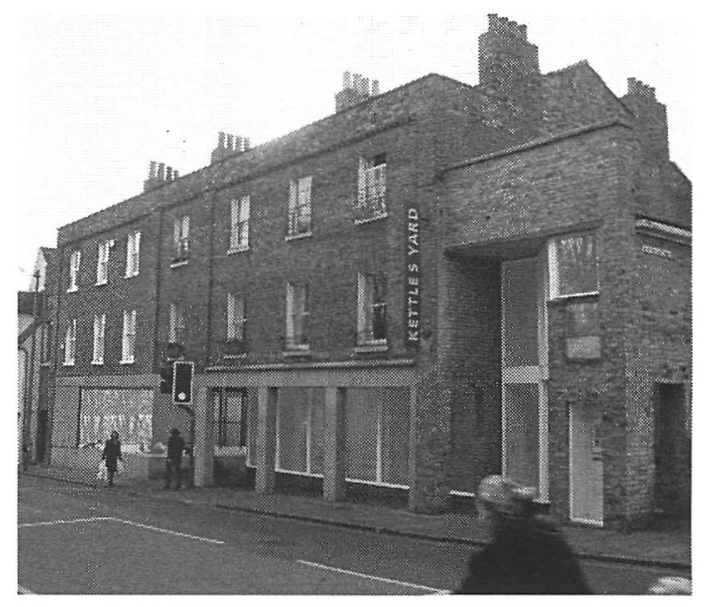

ILL. 5 Vue de la façade victorienne rue Castle pour Kettle's Yard par la firme d'architectes Jamie Fobert de Londres.
[16]

ARNOLD, Bruce. "Kettle's Yard». In. ARNOLD, Bruce. The Art Atlas of Britain and Ireland. Londres: Viking en association avec National Trust, 1991,

p. 183.

[17]

LEWISON, Jeremy. "Ede, Harold Stanley". In. The Oxford Dictionary of National Biography. Oxford et New York: Oxford University Press, vol. 17, 2004, p. 657-658.

[18]

KETTLE'S YARD. Kettle's Yard

House. <http://www.kettlesyard.co.uk/house/index.html>

(consulté en mars 2008).

[19]

ROSE, Cynthia. "Cambridge, Ede's Cornern. ArtNews, vol. 9, novembre 1989, p. 103.

[20]

BARASSI, Sebastiano. "Introduction". Kettle's Yard House Guide. Cambridge: Kettle's Yard, 2002, p. 8.

[21]

LEWISON, op.cit. du Gardner alloué aux expositions temporaires (du 18 septembre 1998 au 3 janvier 1999).

\section{Kettle's Yard}

La deuxième collection fermée que nous présentons est contemporaine au musée Gardner, il s'agit de Kettle's Yard, situéà Cambridge au Royaume-Uni, dont la collection d'art moderne des années 1920 et $1930^{[16]}$ a été assemblée par un collectionneur particulier, Harold Stanley Ede (1895-1990) ${ }^{[17]}$.

La collection renferme des œuvres peintes de Joan Miró, de Christopher Wood et d'Alfred Wallis et des sculptures de Constantin Brancusi, de Barbara Hepworth, d'Henry Moore ${ }^{[18]}$, ainsi que le rassemblement le plus significatif du sculpteur Gaudier-Breska ${ }^{[19]}$. Harold Stanley Ede a travaillé à la Tate Gallery de Londres en tant qu'assistant-conservateur de 1922 à $1936^{(201}$, année de son départ expliqué hypothétiquement en raison du conformisme de l'institution qui rendait difficile l'acquisition d'œuvres contemporaines ${ }^{[21]}$. À l'heure actuelle, Kettle's Yard présente la même problématique puisque sa scénographie est encapsulée à l'intérieur du temps désormais historicisé du collectionneur (le schème d'accumulation de la collection a été réalisé sur une période de 50 ans: de 1923 à 1973) $)^{[221}$. 


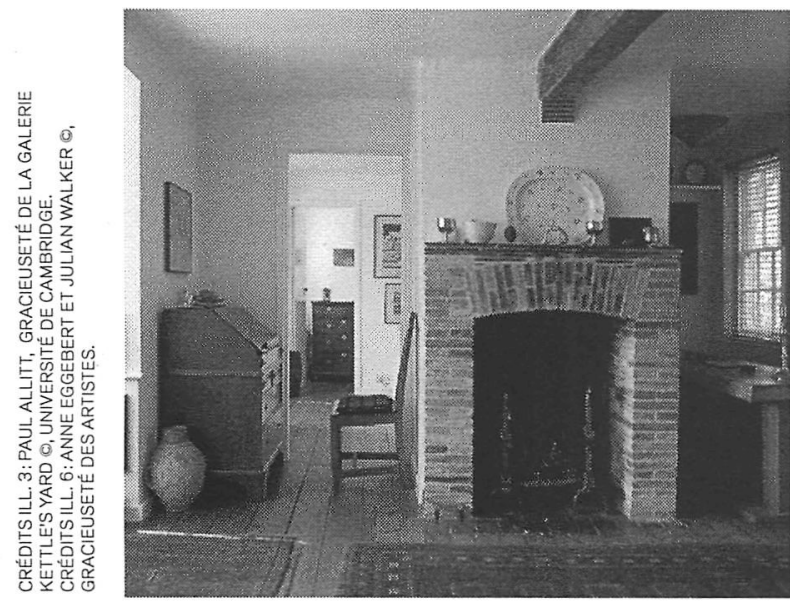

ILL. 3 Le salon de Kettle's Yard.

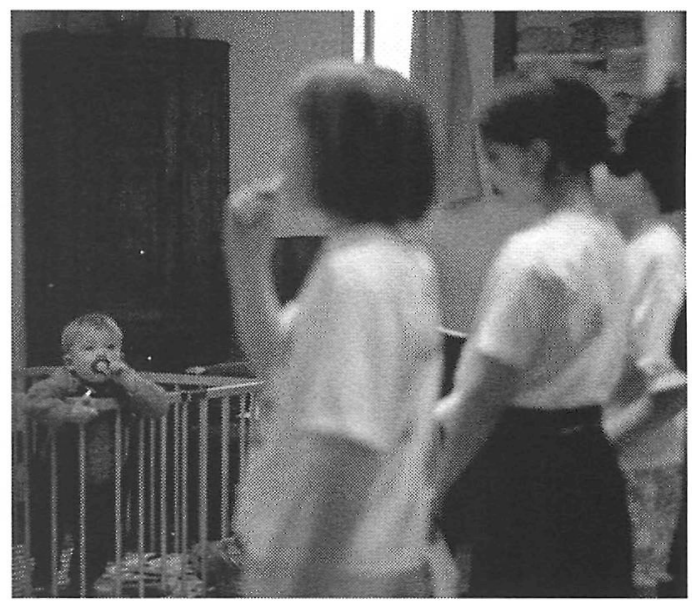

ILL. 6 Mr and Mrs Walker Have Moved - Freddie with Audience, 1998.
Les quatre maisons composant le bâtiment actuel ainsi que la collection ont été légués à l'Université Cambridge en 1966 avec la condition que soient conservés intacts l'environnement domestique et les objets quiy sont distribués ${ }^{[23]}$. Harold S. Ede et sa femme Helen y ont emménagé en 1957 afin d'y vivre et d'agir en tant que guides chaque après-midi pour les visiteurs de la résidence et ce, jusqu'en 1973 .

Kettle's Yard a été l'objet de plusieurs expansions successives (1970 ${ }^{[24]}$, 1980 et $1993 / 1994^{(251)}$ afin de générer des espaces alloués aux expositions temporaires et, tout comme le musée Gardner, l'institution est toujours en cours de financement dans le but d'annexer une nouvelle aile pensée par la firme d'architectes Jamie Fobert, garantissant l'espace qui sera nécessaire aux programmes d'éducation et de conférences ${ }^{[26]}$ (ill. 4 et ill. 5).

Dans le même ordre d'idées que les expositions temporaires réinvestissant le contenu de la collection fermée, le travail d'artistes en résidence permet la création interne de projets questionnant les enjeux directs de l'institution et c'est dans cette perspective que l'œuvre Mr \& Mrs Walker Have Moved des artistes Julian Walker et Anne Eggebert de 1999 s'insère ${ }^{[271}$. Le couple d'artistes et leur fils alors âgé de deux ans ont été invités à demeurer dans le musée pour un séjour d'une semaine afin de tester la véracité de l'environnement domestique de Kettle's Yard (ill. 6).

Les quatre pièces les plus privées du musée (comme les chambres à coucher) ont été capturées par le biais d'une caméra de surveillance en circuit fermé redirigeant les images en direct de la famille à l'intérieur de la galerie d'expositions temporaires (ill. 7). Cette intervention a permis la
[22]

UNIVERSITY REGISTRY.

"Draft, The Glory of the Gardner: Kettle's Yard Gallery", $1^{\text {er }}$ mars 1984. Archives de l'Université Cambridge, "Financial Board records. Ede Benefaction Kettle's Yard", F.B4 19/24 (15 juillet 1983-30 août 1984). [23]

Rob Tufnell et Sebastiano Barassi mentionnent cette entente écrite par le collectionneur dans TUFNELL, Rob. "Sans titre". In. TUFNELL, ROB (dir.). The Unhomely, opuscule de l'exposition du 8 novembre 2003 au 11 janvier 2004. Cambridge (GB): Université de Cambridge, 2003, p. 32; et BARASSI, Sebastiano. "Kettle's Yard. Museum or Way of Life? ". In. SPARKE, Penny (dir.). The Modern Period Room. The Construction of the Exhibited Interior 1870 to 1950. Londres et New York: Routledge, 2006, p. 129.

\section{[24]}

OWERS, David. "Kettle's Yard Design Intentions: What Do We Call the Kettle? ". Cambridge Review, vol. 92, n² 2197, 1970, p. 170. 

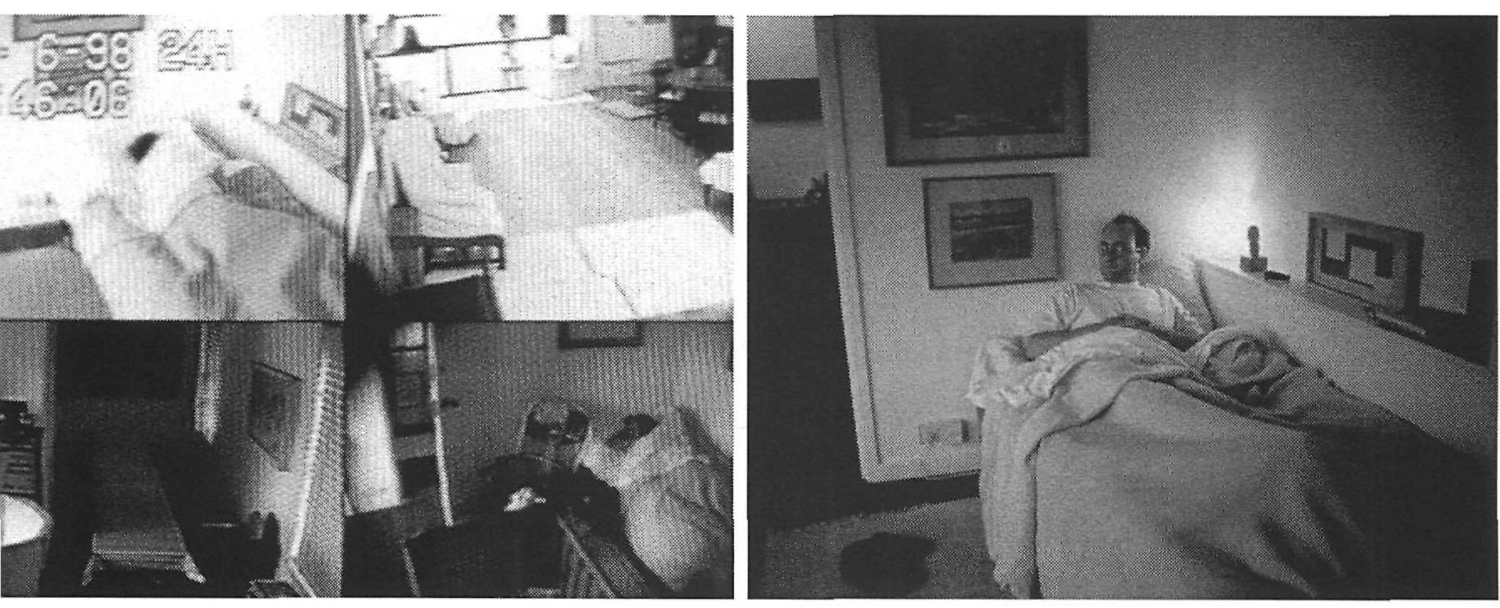

ILL. 7 Mr and Mrs Walker Have Moved - 20-6-98 06:46:06, 1998.

ILL. $8 \mathrm{Mr}$ and Mrs Walker have moved - Julian in Jim's bed, 1998.

[25]

BARASSI, «Introduction", p. 12.

[26]

DORRELL, Ed. «Fobert Looks to the Past for Kettle's Yard Future". The Architects' Journal, vol. 221, $n^{\circ} 7,2005$, p. 7. Pour des plans et des images du projet, voir également JAMIE FOBERT ARCHITECTS. Extensions to Kettle's Yard Gallery. <http// www.jamiefobertarchitects. com/\#/projects/arts/extension_to_kettle's_yard_gallery/> (consulté en juin 2009).

[27]

BARASSI, "Kettle's Yard. Museum or Way of Life?", p. 139.

[28]

PUTNAM, James. Le Musée à l'œuvre, le musée comme médium dans l'art contemporain.

Paris: Thames and Hudson, 2002, p. 174

[29]

VIGURS, Peter F. «Introduction». In. The Garman-Ryan Collection. Illustrated Catalogue. Walsall: W.J. Ray \& Co. Ltd., 1976.

[30]

MOORE, Rowan. «A Pebble on Water». In. MOORE, Rowan et al. The New Art Gallery Walsall. Londres: B.T. Batsford, 2002,

p. 60 . réinscription des paramètres permanents de la collection dans le présent grâce à la remise en question de son aspect narratif par les artistes.

En effet, Julian Walker met en échec l'aspect domestique de Kettle's Yard en exposant l'incommodité de vivre dans le musée/maison ${ }^{[28]}$ et en faisant ressortir son caractère esthétique construit. Cette intervention consiste en une réactualisation puisqu'elle réintroduit la présence de locataires à l'intérieur des lieux privés du musée, réactivant ainsi le temps où il était habité par le propriétaire et sa femme, tout en reconsidérant le contexte premier afin d'en étendre la portée (ill. 8). Mr \& Mrs Walker Have Moved engage également un réaménagement des paramètres temporels du musée par l'utilisation d'une caméra de surveillance qui injecte une temporalité live à son temps immobile et historique. À la manière des photographies d'Abelardo Morell évoquées antérieurement, la redirection et la cohabitation des images de quatre pièces du musée en temps réel sur un écran de télévision engendrent un doublon spatial virtuel et dissemblable du parcours fixe qui réinterprète par le fait même ses notions immuables de lieu. L'histoire fermée de Kettle's Yard se rejoue en direct et propose une vision ubiquiste des lieux d'exposition.

\section{New Art Gallery Walsall}

La New Art Gallery Walsall est une galerie d'art dont la construction est récente en comparaison aux deux autres études de cas : elle a ouvert ses 
portes en février 2000. Situé à Walsall au Royaume-Uni, l'édifice accueille la collection Garman Ryan léguée à la ville en 1972 par deux collectionneuses : Kathleen Garman (1901-1979) et Sally Ryan (1916-1968). Ce rassemblement d'œuvres privées s'est échelonné sur une période de quatorze ans (19591973) ${ }^{[29]}$, privilégiant les œuvres figuratives (la plupart sont des portraits ou des paysages) et celles détenant un caractère inachevé (telles que les esquis-

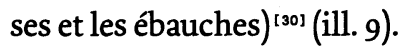

Le site Web de la New Art Gallery Walsall répertorie quelque 365 œuvres dont plus du tiers sont des sculptures issues de différentes périodes et cultures $^{[311}$ et comprend le rassemblement numérique le plus important de l'artiste Jacob Epstein (époux de Kathleen Garman, une des donataires), ainsi que des œuvres peintes et des dessins de petit format d'artistes européens, dont Van Gogh, Monet, Turner, Sisley, Pissarro, Renoir, Corot, Millet ${ }^{\text {[32] }}$. Avant sa redistribution à l'intérieur de la nouvelle construction, la collection appartenait au Walsall Museum and Art Gallery et était installée dans les locaux de la bibliothèque de la ville où elle fut dévoilée pour la première fois en $1973^{[33]}$. Pour sa présentation, Kathleen Garman a personnellement choisi maints détails relevant de l'accrochage, de la couleur des murs et du tapis au sol ${ }^{[34]}$, ainsi qu'un arrangement des œuvres selon des thématiques prédéfinies telles que l'enfance, les animaux, etc. ${ }^{[35]}$. Le document officiel faisant état du don de la collection mentionne certaines restrictions, dont l'interdiction d'aliéner ou de vendre quelque objet que ce soit ainsi qu'une obligation de conserver l'ensemble des objets en tant qu'entité ${ }^{[36]}$.

Afin de construire une nouvelle architecture qui répondrait aux besoins d'une collection particulière de dimension domestique, Adam Caruso et Peter St. John, les architectes de la New Art Gallery Walsall, se sont inspirés du musée Louisiana de Copenhague ainsi que de Kettle's Yard ${ }^{\text {[371. }}$ La résultante de cette étude a été la disposition de la collection à l'intérieur de treize salles ${ }^{[38]}$ de taille réduite dotées d'une fenestration importante et privilégiant la présence accrue de matériaux comme le bois afin d'insister sur le caractère privé de la résidence ${ }^{[39]}$ (ill. 10).

Dans le but de marquer une séparation claire entre le troisième étage destiné aux expositions temporaires et les salles exposant la collection fermée, les architectes ont préconisé des galeries à l'échelle considérablement différente, soit des murs d'une hauteur de six mètres ${ }^{[40]}$.

Puisque aucun ajout ou aliénation d'œuvres n'a pu être réalisé dans le réaménagement de la collection Garman Ryan, l'institution a plutôt opté pour la création de collections parallèles dès 1996 afin d'accumuler conjointement des œuvres contemporaines ou historiques qui permettent une extension critique de la collection fermée. La New Art Gallery Walsall possède maintenant trois autres collections en plus de la Garman Ryan : une

\section{[31]}

Selon la New Art Gallery Walsall, la collection contient majoritairement de l'art européen daté d'après la Renaissance, mais contient également des artefacts grecs classiques, des objets précolombiens, asiatiques, égyptiens et africains. (THE NEW ART GALLERY WALSALL. *A competition to Select an Architect». Archives de la New Art Gallery Walsall, non daté, p. 20.)

[32]

THE NEW ART GALLERY

WALSALL. Collection

Introduction. $<\mathrm{http}: / / \mathrm{www}$.

artatwalsall.org.uk/index2.

asp?sec $=2>$ (consulté en

octobre 2007).

[33]

McGUIRE, Penny. «Rising From

its Industrial Setting, Caruso

St-John's Walsall Gallery is Taking

Cool Britannia to the Country's

Unglamorous Midlands». The

Architectural Record, vol. 189, $n^{\circ}$

5, 2001, p. 217.

[34]

McGREGOR, Sheila. A Shared

Vision: The Garman Ryan

Collection at The New Art

Gallery Walsall. Londres: Merrell Holberton, 1999, p. 43.

\section{[35]}

S'y trouvent les thématiques:

(1) portraits et personnages,

(2) enfants, (3) paysages ruraux

et urbains, arbres, (4) natures

mortes et fleurs, (5) travail et

loisirs, (6) œuvres religieuses

et, finalement, (7) animaux et

oiseaux. Traduction libre des

thématiques du site de LEE,

Chris. Inspiring Creativity Through the Garman Ryan Collection

and the New Art Gallery Walsall.

<http://www.chrislee.org.uk/

InspiringCreativity/Themes.htm> (consulté en mars 2009).

[36]

CLERK, Tom. *Copy Resolutions Passed by the Libraries,

Museums and Art Gallery

Management Committee at their

Meeting Held on $23^{\text {rd }}$ January

1973 x. Archives de la New Art

Gallery Walsall. 


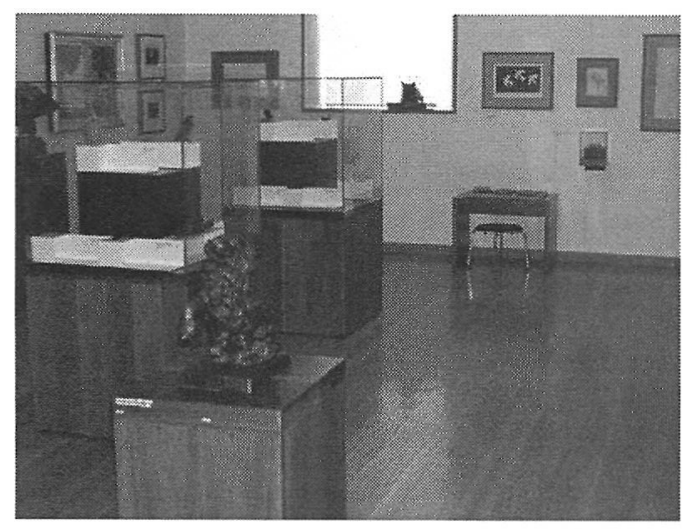

ILL. 9 La collection Garman Ryan.

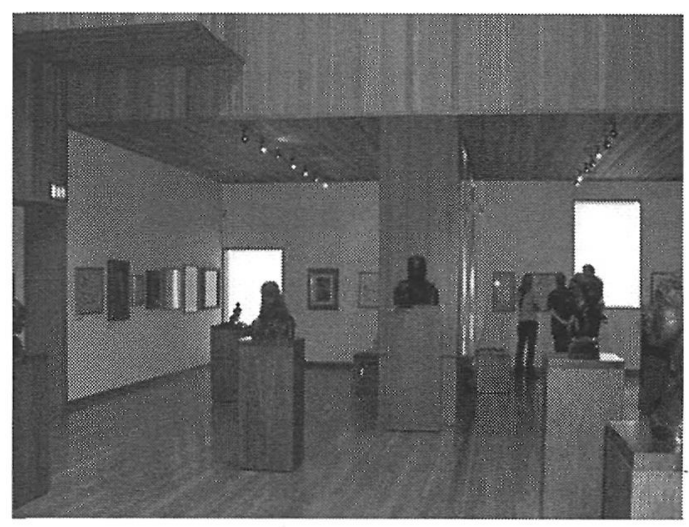

ILL. 10 La collection Garman Ryan de la New Art Gallery Walsall.
[37]

BANN, Stephan, "A Way of Life: Thoughts on the Identity of the House Museum ". In. PAVONI, Rosanna (dir.). Historic House Museums Speak to the Public: Spectacular Exhibits Versus a Philological Interpretation of History. Actes de la conférence annuelle du DEMHIST, Genève, 1-4 novembre 2000, p. 25-26.

[38]

PEARMAN, Hugh. «Youthful London Duo Builds an Unusual Art Gallery in an Out-of-the-Way Location". Architectural Record, vol. $188, n^{\circ} 4,2000$, p. 32.

[39]

CARTER, Brian. «Solid Citizen».

The Architectural Review, vol. 207, n 1239, 2000, p. 66. Voir également CARUSO \& ST. JOHN. New Art Gallery and Square, Walsall, UK, 1995-2000. $<$ http:/www.carusostjohn.com/ www/flash.htm $\triangleright$ (consulté en novembre 2007).

[40]

CARTER, p. 66.

[41]

THE NEW ART GALLERY WALSALL, Collection Introduction, op. cit. collection permanente consistant en un amalgame d'objets amassés depuis 1892, la collection Garman Ryan Epstein, ainsi que la collection d'archives Epstein jointe aux services de la bibliothèque.

L'analyse rapide des politiques d'acquisition de la collection parallèle Garman Ryan Epstein ${ }^{[41]}$ nous permet d'affirmer qu'une critique et un réajustement des lacunes de la collection principale sont souhaités ${ }^{[42]}$. Ces politiques mentionnent en effet que l'acquisition d'œuvres supplémentaires de Jacob Epstein sera envisagée si elles exhibent des aspects de sa pratique qui sont sous-représentés ${ }^{[43]}$ dans la collection fermée, transformant la New Art Gallery Walsall en un centre d'études de premier ordre sur l'artiste ${ }^{[44]}$. Le règlement d'acquisition démontre également son intérêt par rapport aux œuvres facilitant la médiation de la collection privée. Enfin, les politiques d'acquisition stipulent que l'inclusion d'œuvres d'artistes non représentés par la collection Garman Ryan, mais dont la pratique se relie, de manière formelle ou thématique à celle du sculpteur Jacob Epstein, sera envisagée ${ }^{[45]}$.

\section{De la collection à l'exposition}

En terminant, l'extraction des stratégies de réactualisation identifiées sur les trois collections fermées a également fait apparaître un " nouveau " rôle de l'intervenant interne du musée (directeur, conservateur des collections, commissaire d'exposition, etc.). Manifestement, celui-ci parvient à instaurer une distance critique par rapport au nucléus fermé de la collection grâce à la redistribution des énergies de l'institution vers des projets temporaires et 
contemporains qui offrent une marge de manœuvre difficile à obtenir dans la seule mise en valeur de l'assemblage permanent toujours sous l'emprise des restrictions du donateur originel. Cette tendance générale a été notée à plusieurs reprises, notamment par Francis Haskell dans son ouvrage Le musée éphémère ${ }^{[46]}$ :

Nul n'aurait pu prédire le changement survenu dans la seconde moitié du XXe siècle, lorsque la quasi-totalité des grands musées et galeries du monde se mirent, l'un après l'autre, à organiser ou à accueillir de telles expositions [l'auteur traite des grandes expositions temporaires de type blockbuster]. Aujourd'hui, dans l'esprit des visiteurs, ces institutions sont autant associées aux expositions temporaires qu'à leurs collections "permanentes ". Les institutions plus modestes demeurées fidèles au veto explicite de leurs fondateurs concernant le prêt d'œuvres d'art ont été soumises à de fortes pressions : dans les années récentes, la fondation Barnes de Philadelphie et la fondation Burrell de Glasgow se sont ainsi vues contraintes de changer de politique. Les administrateurs de la collection Wallace n'ont résisté qu'avec difficulté au chantage et aux intimidations des grandes institutions, qui partageaient jadis les mêmes idéaux ${ }^{[47]}$.

Ce contexte en transformation rend de plus en plus difficile la gestion des demeures historiques-musées et des collections fermées qui doivent s'adapter aux nouvelles exigences des visiteurs (nous pensons notamment à l'ajout de restaurant, de café, de boutique, de hall de réception et d'accueil, d'ascenseur, etc.) et à une distribution des énergies de plus en plus grande vers le secteur contemporain. Finalement, nous espérons que notre étude mettra en évidence la possibilité d'un juste milieu entre la conservation intégrale de la collection (aucune intervention directe) et les changements radicaux (notamment par la ratification du testament du collectionneur).
[42]

«The collection will seek to build on the existing Garman Ryan Collection, filling gaps where they exist, but also building on its spirit and creative impetus. " (MUSEUMS, LIBRARIES, ARCHIVE COUNCIL, MUSEUMS LIBRARIES ARCHIVE COUNCIL. *The Garman Ryan Collection *, Discovering UK Collections. <http:// www.cornucopia.org.uk/html/ search/verb/GetRecord/454> (consulté en novembre 2007). [43]

À ce titre, il est indiqué que la collection ne comprend aucune œuvre sculptée dans la pierre, négligeant un aspect primordial du cheminement de l'artiste. (THE NEW ART GALLERY WALSALL. «A competition to Select an Architect », p. 25.)

[44]

THE NEW ART GALLERY WALSALL. The Garman Ryan Epstein Collection - Collection Policy. <http://www.artatwalsall.org.uk/index2.asp?sec $=2>$ (consulté en septembre 2007). [45]

Id.

[46]

HASKELL, Francis. Le musée éphémère: les maîtres anciens et l'essor des expositions [trad. de l'anglais Pierre-Emmanuel Dauzat]. Paris: Gallimard, coll. "Bibliothèque des histoires", 2002, 261 p. Ce sujet particulier est également couvert dans HASKELL, Francis, L'amateur d'art Paris: Librairie générale française, coll. "Livre de Poche, Références», 1997, 351 p.

[47]

Id., p. 189. 
Updating the closed collections, three case studies. Collections from the Isabella Stewart Gardner Museum, the Kettle's Yard, and the Walsall New Art Gallery

[Translated by Philippe Lagrange]

Our study takes place in a context where, in many cities, mostly in the United States and Europe (France, United Kingdom, Netherlands, Italy), donators have offered private collections that were limited by various provisions of the will. These collections are considered closed, because any modification of the objects composing them would result in the loss of the ownership rights. Even though they are slowly turning from a domestic environment into a public museum, these places are still reflecting their original owner's idiosyncrasies and still manage to keep their "private character."

Over the last two decades, a new trend developed among the institutions who manage that kind of closed collections. Museum curators began developing projects that allowed intervening in these collections without derogating from the laws that maintain them closed.

The first objective of this research is to define the so-called "closed collection" in order to address four kinds of interventions that allow an update of a closed collection structure, and at the same time ensure its physical integrity. These methods were extracted from three case studies: the Isabella Stewart Gardner Museum in Boston, Kettle's Yard in Cambridge, and the New Art Gallery in Walsall. (1) The first approach is the adjacent construction or the retrofitting of the interior of a pre-existing pavilion. (2) In the second one, the spaces recently created allow the presentation of temporary expositions (historical or contemporary), thematically or formally related to other aspects of the permanent collection. (3) The third one consists in facilitating the introduction of artists on site and promoting creative work complementary to the closed collection. (4) Finally, accumulating supplementary artworks as components of parallel collections brings a balance to the main collection's content by filling in the gaps or by demonstrating extrapolations. 
To conclude this article, we will hypothetically demonstrate that these interventions should be taken as critics rather than as a valorization of the collection's wealth and also that these interventions allow a macroscopic thinking about the actual changes taking place in the museum-related world. 